\title{
Fertility-Preserving Treatments in Patients with Gynecological Cancers: Chinese Experience and Literature Review
}

\author{
Chun-Yan Liu*, Hua-Jun Li, Hua Lin, Bin Ling
}

\begin{abstract}
We conducted a retrospectively reviewed of the literature published of patients underwent fertility-preserving treatments for cervical, endometrial and ovarian cancers using the WANFANG database in Chinese. A majority were retrospective studies and case reports. With cervical cancer, radical trachelectomy $(R T)$ in combination with pelvic lymphadenectomy could preserve the fertility of patients with early stage IA1-IB1 cancers, Tumor size $\leq 2 \mathrm{~cm}$ should be emphasized as the indication of RT in considering of the higher recurrent rate in patients with tumor size $>\mathbf{2} \mathbf{c m}$. For endometrial cancers, there is much experience on it. Given accurate pretreatment assessment, hormonal therapy is feasible management option to preserve fertility in young patients with early stage lesions that limited to the endometrium and well differentiated. High dose progestin have been applied, oral medroxyprogesterone acetate (MPA), $250-500 \mathrm{mg} / \mathrm{day}$, megestrol acetate $160-480 \mathrm{mg} / \mathrm{day}$. Other therapies that have been used in a limited number of cases include GnRH analog, intrauterine devices (IUDS) containing progestogen, usually combination of these therapies. All patients should be followed up by ultrasound and/or MRI evaluation, and endometrial curettage at intervals of 3 months. With ovarian cancer, in China, fertilitypreserving surgery in patients with stage IA (grade G1) of epithelial ovarian tumor and patients with germ cell tumor and borderline ovarian tumor have been successfully performed.
\end{abstract}

Keywords: Gynecological cancers - fertility preserving - treatment - recurrence - outcome

Asian Pac J Cancer Prev, 16 (12), 4839-4841

\section{Introduction}

In recent years, fertility-preserving is rapidly expanding new field. Cancer may be detected at any age, thousands of reproductive-age women and girls are diagnosed with cancer every year (Howlader et al., 2014). Today Reduction of cancer-related mortality remains the main issue of the health-care provider, but the fertilitypreserving ability in the increasing population of young and delaying childbearing women with gynecological cancers also is considerable. Fertility-preserving treatments have been successfully performed in early stage cases of cervical, endometrial and ovarian cancers, although the awareness and access to fertility-preserving was limited in the beginning (Mojgan Karimi Zarchi et al., 2011). Here, we report our present experience of fertilitypreserving treatments in gynecological cancers and focus on new developments on the horizon in the literature.

\section{Cervical Cancer}

Cervical cancer is still the most common gynecological cancer in the world despite the popularization of screening program for cervical cancer and the adoption of vaccination against human papilloma virus(HPV) in many country (Jeong-yeol et al., 2015). In China, as the nationwide of screening for cervical cancer and releasing of family-plan, the young person with early-stage of cervical cancer is increasing, many of them desire for producing and raising own children.

The fertility-preserving treatment of Ia1 and Ia2 cervical squamous cell carcinoma is conization, which is conservative used only in cervical adenocarcinoma Ia1 (Yang et al., 2013). According to study, the procedure, such conization, is successfully therapy for pre-invasive lesions and some microinvasive carcinoma (limited to $\leq 3 \mathrm{~mm}$ ) without lymphovascular invasion (Baalbergen et al., 2011). The risk factor of recurrence or remains for conization is positive margin, lymphovascular invasion and multi-center lesions. In order to avoid remains, it is important that to choose appropriate range of conization. It is necessary that the width of conization is $0.3 \mathrm{~cm}$ outside of the lesions, and the depth of conization is $2.0-2.5 \mathrm{~cm}$ of cervical canal. The microinvasive cervical carcinoma with positive-margin, it is recommended that it is coned again or managed as stage Ib1 according to FIGO guideline for cervical cancer. But the treatment of stage Ia1 with lymphovascular invasion and stage Ia2 of cervical cancer is not only conization, but also total pelvic lymphadenectomy should be performed.

Radical trachelectomy (RT) is defined as removal of cervix and parametrium, preserving ovaries and uterus 
body and grafting uterus body to vagina at the end of the operation. RT could be completed through vaginal, abdominal and laparoscopically way, which is good option to preserve fertility meanwhile threatment of cancer (Dargent et al., 1994; 2000; Li et al., 2011; Chen et al., 2013; Yao et al., 2013; Cao et al., 2013).

Selection criteria for RT were as below: young patients desire for fertility without infertility factors, Tumor size $\leq 2 \mathrm{~cm}$, tumor stage Ia2-Ib1 without lymphovascular invasion, endocervix upper intact. Some believed that It is recurrence easily after RT if Tumor size is $>2 \mathrm{~cm}$, tumor stage is $\mathrm{Ib} 2$ or higher with lymphovascular invasion (Dargent et al., 1994) . It is very important to evaluate tumor size, stage, lymphovascular and endocervix upper invasion or not, which can be measured aided through MRI and colposcopy before procedure.

Early detection of recurrent may impact not only survival of patient but also fertility wish undergo RT. Close follow-up is necessary in any patient after RT treatment. Follow-up should be done every month in first half year after RT, including: gynecological check, ultrasound, and serum SCC-Ag, CT, MRI and PET-CT at necessary, every 2 month for the last half year, then every 3 month for 3 years, and every half year after that. Cytology evaluation of vaginal vault should be done every 3 month, fertility could be suggested with twice negative cytology evaluation.

\section{Endometrial Cancer}

The number of endometrial cancer is increasing trend as the changing of life style and diet structure in Chinese women. It has been proving that the treatment of high effective progestin is good option for young person with endometrial cancer (Chiva et al., 2008; Dursun et al., 2012; Yu et al., 2009; Wang et al., 2014).

Complex atypical hyperplasia of endometrium is a precursor of endometrial adenocarcinoma, which is the most common histological type of endometrial cancer, although the ultimate treatment is surgery in early-stage lesions for them, hormonal therapy such as progestin has been suggested to young women age $\leq 40$ who anxious to conserve their fertility (Yu et al., 2009; Wang et al., 2014). Besides, Selection criteria for progestin therapy have to be content with things as high grade, lesion limited to the location in the endometrium without involvement of myometrium, lymph node or the cervix. The express of progestin receptor is positive and patient's consent must be done fully before conservative therapy.

The evaluation before progestin therapy for early-stage endometrial cancer should be done, including: history of disease, check-up, all body status, Pathological review and the severe degree of lesion through ultrasound or MRI (Cade et al., 2010), PET-CT at necessary. The method of therapy is high dose progestin used, oral medroxyprogesterone acetate (MPA), 250-500mg/day, megestrol acetate $160-480 \mathrm{mg} /$ day. Other therapies that have been used in a limited number of cases include GnRH analog, intrauterine devices (IUDS) containing progestogen, usually combination of these therapies. The evaluation of curative effect is very important. All patients should be followed up by endovaginal ultrasound and/or MRI evaluation, and endometrial curettage at intervals of 3 months. The criteria of judgment for therapy end-point is divided by remission completely, remission partially, no response to progestin therapy or disease stable and deteriorate. The indication of termination for progestin therapy is one of them: disease deteriorate, no desire for fertility, remission completely, severe side-effect and no response to progestin therapy after six months.

For those patients with remission completely, the therapy purpose is to maintain normal period and prevent recurrence, if they have no plan for fertility in short-time, While the therapy purpose is to monitor ovulation and complete fertility as soon as possible for the patients who anxious to conceive and give birth to a child.

\section{Ovarian Cancer}

The highest mortality is caused by ovarian cancer in gynecological malignant tumor. They have different clinical expression in different type of ovarian cancer, so do different manage and prognosis. The decision of preserving-fertility treatment for patients with ovarian cancer depends on age, type and stage (Liu et al., 2013).

Epithelial ovarian cancer (EOC) is the most common histological type. Although most of them are diagnosed in postmenopausal women, 3-17\% of EOC occurs in women under 40 years old and less than 30 years (Plaxe et al., 1993; Rodriguez et al., 1994; Schilder et al., 2002; Park et al., 2008; Anchezar et al., 2009). We need take discreet attitude to perform preserving-fertility surgery for women with EOC. To weigh the advantages and disadvantages, explain them to patients, and sign medical informed consent (MIC) before preserving- fertility surgery taken. Some criteria must be satisfied as below: young patient age $\leq 35$ who desire to conserve their fertility, stage Ia, G1, the opposite ovary with good appearance and negative pathology, negative abdominal cavity cytology and lymph node in high-risk district, Compliance with follow-up.

But in malignant ovarian germ cell tumor (GCT), unilateral salpingooophorectomy with preservation of the contral lateral ovary and uterus is considered the appropriate surgical treatment to conserve fertility, without regarding to stage, even in the case of advanced germ cell tumor, particularly if the contra lateral ovary is normal (Ghaemmaghami et al., 2010; Wang et al., 2011; Liu et al., 2013), considering most GCT is unilateral ovary disease, sensitive to chemotherapy cisplatin+ etoposide + bleomycin (PEB) and cisplatin+ vincristine + bleomycin (PVB). For early-stage ovarian dysgerminoma and high differentiation ovarian immature dermoid tumor, preserving- fertility staging surgery including omentectomy and retroperitoneal lymph nodes dissection.

For young patient age $\leq 40$ with borderline ovarian tumor, unilateral salpingooophorectomy with preservingfertility is recommended, Staging surgery is not be suggested for them, considering pelvic cavity adhesion is caused by it (Wang et al., 2011). Bilateral borderline ovarian tumors is less happened, we can only perform removing the tumors with ovaries left as long as normal ovarian tissue existing. Preserving-fertility therapy is 
adapted for advanced borderline ovarian tumor without contral lateral ovary and uterus involved and negative exogenous nipple structure and Invasive plant. Patients with borderline ovarian tumor are normally young with recurrent character easily after surgery, so provider should explain the advantage and disadvantage of Preservingfertility therapy, sign informed consent.

\section{Conclusion}

Preserving-fertility treatment should be suggest for young patients with gynecological cancers who desiring future childbearing, but we should address the possibility of infertility with patients treated before cancer treatment, the discussion and informed consent should be documented. If any potential threats to fertility in the treatment process happened, the patient should be encouraged to consider consulting reproductive specialists. Sperm and embryo cryopreservation as well as oocyte cryopreservation are considered mature practice and widely available (Gurgan et al., 2008; Cao et al., 2009; Noyes et al., 2009; Cobo et al., 2011), ovarian tissue cryopreservation is only option for children without recourse to ovarian stimulation and sexual maturity. Other Preserving-fertility methods should be further investigated without recurrence danger.

\section{References}

Anchezar JP, Sardi J, Soderini A (2009). long-term follow-up results of fertility sparing surgery in patients with epithelial ovarian cancer. $J$ Surg Oncol, 100, 55-8.

Baalbergen A, Smedts F, Helmerhorst TJ (2011). Conservative therapy in microinvasive adenocarsinoma of the uterine cervix is justified: an analysis of 59 cases and a review of the literature. Int J Gynecol Cancer, 21, 1640-45.

Cade TJ, Quinn MA, McNally OM, et al (2010). Predictive value of magnetic resonance imaging in assessing myometrial invasion in endometrial cancer: is radiological staging sufficient for planning conservative treatment? Int Gynecol Cancer, 20, 1166-69.

Cao DY, Yang JX, Wu XH, et al (2013). comparisons of vaginal and abdominal radical tracheletomy for early-stage cervical cancer: preliminary results of a multi-center research in China. Br J Cancer, 109, 2778-82.

Cao Y, Xing Q, Zhang ZG, et al (2009). Cryopreservation of immature and in-vitro matured human oocytes by vitrification. Reprod Biomed Online, 19, 369-73.

Chen Yi-le, Li Le-sai, Tang Zhen-zi, et al (2013). Analysis of the security, pregnancy outcomes, and the tumor recurrence related factors of young patients with cervical cancer treated with different radical trachelectomy. Chin J Obstet Gynecol, 48, 352-57.

Chiva L, Lapuente F, Gonzalez-Cortijo L, et al (2008). Sparing fertility in young patients with endometrial cancer. Gynecol Oncol, 111, 101-104.

Cobo A, Diaz C (2011). Clinical application of oocyte vitrification: a systematic review and meta-analysis of randomized controlled trials. Fertil Steril, 96, 277-85.

Dargent D, Brun JL, Roy M, et al (1994). Pregnancies following radical trachelectomy for invasive cervical cancer. Gynecol Oncol, 52, 105.

Dargent D, Martin X, Sacchetoni A, et al (2000). Laparoscopic vaginal radical trachelectomy: a treatment to preserve the fertility of cervical carcinoma patients. Cancer, 88, 1877-82.
Dursun P, Erkanli S, guzel AB, et al (2012). A Turkish Gynecologic Oncology Group study of fertility-sparing treatment for early-stage endometrial cancer. Int J Gynaecol Obstet, 119, 270-73.

Ghaemmaghami F, Karimi Zarchi M, Naseri A, et al (2010). Fertility sparing in young women with ovarian tumors. Clin Exp Obstet Gynecol, 37, 290-94.

Gurgan T, Salman C, Demirol A (2008). Pregnancy and assisted reproduction techniques in men and women after cancer treatment. Placenta, 29, 152-59.

Howlader N, Noone AM, Krapcho M, et al (2014). SEER cancer statistics review, 1975-2010. National Cancer Institute, HTTP://seer.cancer.gov/csr/1975_2010/.

Jeong-Yeol Park, Hextan Yuen Sheung Ngan, Won Park, et al (2015). Asian society of gynecological oncology international workshop 2014. J Gynecol Onco, 26, 68-74.

Li J, Li Z, Wang H, et al (2011). Radical abdominal tracheletomy for cervical malignancies: surgical, oncological and fertility outcomes in 62 patients. Gynecol Oncol, 121, 565-70.

Liu Q, Ding X, Yang J, et al (2013). The significance of comprehensive staging surgery in malignant ovarian germ cell tumors. Gynecol Oncol, 131, 551-54.

Mojgan Karimi Zarchi, Azamsadat Mousavi, Mitra Modares Gilani, et al (2011). Fertility sparing treatments in young patients with gynecological cancers: Iranian experience and literature review. Asian Pac J Cancer Prev, 12, 1887-92.

Noyes N, Porcu E, Borini A (2009). Over 900 oocyte cryopreservation babies born with no apparent increase in congenital anomalies. Reprod Biomed Online, 18, 769-76.

Park JY, Kim DY, Suh DS, et al (2008). outcomes of fertilitysparing surgery for invasive epithelial ovarian cancer: oncologic safety and reproductive outcomes. Gynecol Oncol, 110, 345-53.

Plaxe SC, Braly PS, Freddo JL, et al (1993). Profiles women age 30-39 and age less than 30 with epithelial ovarian cancer. Obstet Gynecol, 81, 651-54.

Rodriguez M, Nguyen HN, Averette HE, et al (1994). National survey of ovarian carcinoma XII. Epithelial ovarian malignancies in women less than or equal to 25 years of age. Cancer, $\mathbf{7 3}, 1245-50$.

Schilder JM, Thompson AM, DePriest PD, et al (2002). outcome of reproductive age women with stage IA or IC invasive epithelial ovarian cancer treated with fertility-sparing therapy. Gynecol Oncol, 87, 1-7.

Wang Li-ping, Qiao Juan, Li Hai-ye, et al (2014). The effect of fertility-preservation therapy for 16 cases with endometrial carcinoma. Chinese Clin Oncol, 19, 164-67.

Wang Yan, Wang Yue, Li Yi, et al (2011). Investigation of the status and recurrence of young non-benign ovarian tumor patients after fertility preservation therapy. Chin J Clin Obstet Gynecol, 12, 425-27.

Yang Fan, Wang Yue, Zhao Chao, et al (2013). Oncological safety and pregnancy after Cold-knife conization in earlystage cervical cancer. Chin J Clin Obstet Gynecol, 14, 8-10.

Yao Yuan-yang, Wang Yue, Wang Jian-liu, et al (2013). A multicenter study on the outcomes of the oncology, fertility and pregnancy in patients after undergoing the fertility-sparing treatments with early-stage cervical cancer. Chin J Clin Obste Gynecol, 14, 4-7.

Yu M, Yang JX, Wu M, et al (2009). Fertility-preserving treatment in young women with well-differentiated endometrial carcinoma and severe atypical hyperplasia of endometrium. Fertil Steril, 92, 2122-24. 